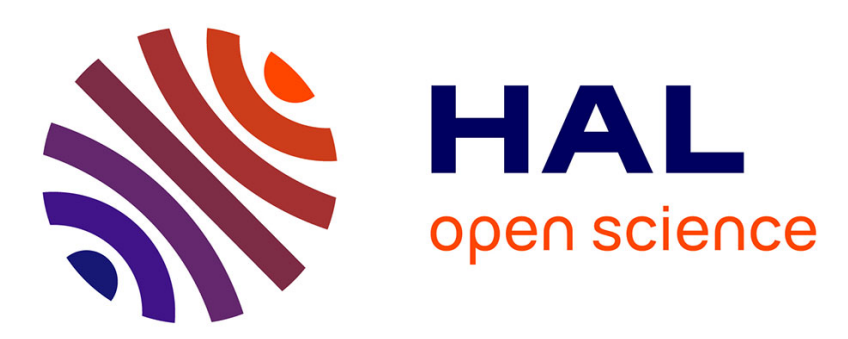

\title{
A Final Cure to the Tribulations of the Vacuum in Quantum Theory
}

\author{
Joseph J Jean-Claude
}

\section{To cite this version:}

Joseph J Jean-Claude. A Final Cure to the Tribulations of the Vacuum in Quantum Theory. 2019. hal-01991699

\author{
HAL Id: hal-01991699 \\ https://hal.science/hal-01991699
}

Preprint submitted on 24 Jan 2019

HAL is a multi-disciplinary open access archive for the deposit and dissemination of scientific research documents, whether they are published or not. The documents may come from teaching and research institutions in France or abroad, or from public or private research centers.
L'archive ouverte pluridisciplinaire HAL, est destinée au dépôt et à la diffusion de documents scientifiques de niveau recherche, publiés ou non, émanant des établissements d'enseignement et de recherche français ou étrangers, des laboratoires publics ou privés. 


\title{
A Final Cure to the Tribulations of the Vacuum in Quantum Theory
}

\author{
Joseph J. JEAN-CLAUDE \\ April 16, 2017 - Revision I (Jan-2019) C Copyright \\ quantogeometry@gmail.com
}

\section{Abstract}

Quantum Field Theory seems to be of late anything but unraveling, apparently shifting from crisis to crisis. Undeniably, there seems to be running, for many different reasons, a rampant discomfort with the Standard Model, an extension of QFT aiming at complementing it. Prior to the latest supersymmetry upset, what has been known as the "vacuum castastrophe" or the "cosmological constant problem" resoundingly shook the very foundations of the Theory. Effectively the enormous numeric disparity between its predicted value of the vacuum energy density, an element of utmost significance in the Theory, and the measured value of this quantity from a Relativistic approach has prompted some to qualify this mishap as the biggest predictive failure of any Theory. Like a wounded beast, Quantum Field Theory seems to be left searching for its soul now for almost two decades. Under the umbrella of QuantoGeometric Theory, we offer here a different approach to the question of the vacuum energy density that demonstrably calculates a sensible and consistent value of this quantity from first axiomatic principles, casting a figure that is in very close match with the cosmological quantity. Therefore in so doing, Quanto-Geometric Theory effectively and definitively cures the long-standing grueling vacuum problem by bridging the gap between the quantum vacuum and the relativistic vacuum.

Keywords: mathematical physics, theoretical physics, quantum field theory, quanto-geometry, quanto-geometric theory, quantum mechanics, momentum space, vacuum energy density, Hilbert space, shells of matter, world planes, omniverse, cosmological constant problem, vacuum catastrophe

\section{Introduction: The Vacuum Catastrophe}

If by many regards, A. Einstein's theories of Relativity are incompatible with the Quantum Theory, it is in the question of characterization of the vacuum that both theories have proven to be dramatically irreconcilable with one another, to the consternation of one generation of physicists after another.

While some physicists such as Leonard Susskind views the enormous disparity in the values upheld by each of the two theories for the vacuum energy density as a "cataclysmic" event figuratively, Nobelist Frank Wilczek (2001) has characterized the deep-reaching implications of the situation in the following terms [6]:

"We do not understand the disparity. In my opinion, it is the biggest and most profound gap in our current understanding of the physical world. ... [The solution to the problem] might require inventing entirely new ideas, and abandoning old ones we thought to be well-established. ... Since vacuum energy density is central to both fundamental physics and cosmology, and yet poorly understood, experimental research into its nature must be regarded as a top priority for physical science."
Without a doubt, the wildly differing values of the vacuum energy density implies serious flaws in the reasoning involved whether in one or both analytic frameworks in use for our understanding of the quantum realm and the cosmological realm. The methods used in quantum theory for the determination of the vacuum energy density are several and remain purely theoretical, whereas in relativistic cosmology astrophysical observations and analyses play a major role in arriving at what appears to be the most consistent empirically-based determinations of the vacuum energy density. Because the notion of vacuum energy density implies an understanding of the very ultra-structure of the vacuum, which Quantum Field Theory is intimately concerned with, the error in the value of the quantity almost constitutes a blanket indictment to the postulates and methods of the Theory.

\section{Quantum Theory View of the Vacuum: a Series of Wrong Assumptions}

High energy physics (HEP) considers that the vacuum contains many juxtaposed fields and can be modeled in terms of quantum harmonic oscillations occurring at each point of the fields constituting the fabric [15]. To evaluate the HEP vacuum energy density the analyst assumes as a matter of course that there is a particle in each unit volume of the vacuum, commonly defined as the cube of the applicable Compton wavelength $\left(\lambda_{C}^{3}\right)$. 
In order to avoid an "ultraviolet divergence" caused by a potentially infinite sum of continual fields's vacuum energy densities, a cutoff to the appropriate energies and wavevectors of the vacuum fluctuations is required. The most common assumption regarding this cutoff is that the putative Planck scale determines the most appropriate ultraviolet cutoff limit for calculating the HEP vacuum energy density. This gives us the vacuum energy density as evaluated below:

$$
\rho_{\text {hep }}=\frac{m_{P}^{4} c^{3}}{h^{3}}=2.44 \cdot 10^{91} \mathrm{~g} / \mathrm{cm}^{3}
$$

where $\rho_{\text {hep }}$ is the vacuum energy density of high energy physics, $m_{P}$ is the Planck mass, $c$ is the speed of light and $h$ is the Planck constant. This number is 122 orders of magnitude bigger than the one straightforwardly cast in relativistic cosmology, which is:

$$
\rho_{\cos }=0.8 \cdot 10^{-29} \mathrm{~g} / \mathrm{cm}^{3}
$$

There are many more methods of calculating this quantity in QFT, which we invoke later, all of which cast vastly differing values to the cosmological value. It requires a lot of selfindulgence, if not motivated blindness, on the part of modernday quantum theoreticians involved in this matter not to firstly and directly view the so-called vacuum catastrophe as an indictment of the Normalization procedure along with its siblings, an illicit mathematical method vehemently decried by P. M. Dirac and R. Feynman at the very inception period of the Theory [13].

I have to insist that in discussing the Quantum Theory in any conference one always has to make an unmitigated distinction between good old Quantum Mechanics and the gauge theory constructions that have been thrown on top of it for many different reasons, the HEP Standard Model being the most accomplished form of these constructions.

In itself, the fact that we have to resort to disallowed mathematical procedures to assert our views about nature's quantum physics should teach us that these views must be either significantly or at least partially wrong. The "cataclysmically" wrong value of the vacuum energy density is there to indict those views, let alone compel us to surgically remove the Normalization procedure as an analytic method from the Theory, and all theories for that matter.

\section{QFT's Ignorance of Ontological Nature of the Vacuum}

The idea that the vacuum is a tapestry of points, whether these points are assumed to be real or virtual particles, is patently wrong and goes against A. Einstein's visualization of the vacuum as a "zero-point energy field"[9]. The discrepancy between the two views starts there indeed. The vacuum is everything that a scalar point is not, in the sense that they are mathematically orthogonal to one another, per David Hilbert's function spaces [3]. Understandably driven by the notion of discrete energy packets, currency of the Quantum Theory, in applying this notion to the tapestry of the vacuum, quantum theoreticians have been led to construct a vacuum that is an expression of scalar packets coming into and out of existence in exotic jostling fluctuations amidst its fabric. In other words, they cannot conceive of a vacuum that is purely and simply empty. They contend that it is not empty, while A. Einstein contented instead that it is an empty continuum, which drove him to ingeniously model it with differential geometry tensors.

What we have done in Quanto-Geometric Theory is to augment the continuum approach to its highest possible level of genericity by going beyond the gravitational properties of the vacuum. That elevation gives us the required distance to decisively settle the debate, because it allows us to derive the fundamental numbers that scaffold the tapestry of the vacuum, both the quantum and the cosmological vacua.

Furthermore, the Quanto-Geometric view proposes a revision of the notion of physical symmetry which is essential in a complete theory of the vacuum meant to encompass the entire scale set that nature has put before us in the 3dimensional realm, from the quantum to the cosmological.

\section{Shells of the Vacuum}

If the putative Planck scale is useful in any ways, for a characterization of the vacuum it certainly looms as the most inappropriate scaling measure to be applied to its tapestry. There is no possible quantization of the vacuum if it must remain true to itself as a continuum. So therefore it cannot be a network of spins or strings because such elements would break it up in discrete parts.

Gauge theories have laudably attempted to otherwise approach the vacuum in terms of potentials, which is admittedly an improvement over discretisation. The problem with the notion of potentials is too much vagueness for an ontological definition. That is the very reason why calculational methods arising from the concept of vacuum potentials are only able to become sensible only when two potentials are considered but not just one, specifically a 
difference of potentials. In that sense the potentials only have a quantitative value but not a qualitative one. Admittedly, vacuum permittivity $\left(\varepsilon_{0}\right)$ and permeability $\left(\mu_{0}\right)$ do not constitute ontological characterizations of the vacuum as firstorder potentials, but artificial constructions whose values have been expressly chosen to satisfy the relation:

$$
c=\frac{1}{\sqrt{\varepsilon_{0} \mu_{0}}}
$$

The vacuum of Gauge theories has been extensively shaped by this model however, that is to say, Maxwell's theory of electromagnetism [15].

Gauge theory model of the vacuum is notably unable to establish a distinction between the vacuum innate to i.e. an elementary particle, from that innate to an atom or a molecule, or the vacuum spread within a solar system, and so on and so forth. In gauge theories, the vacuum is the same everywhere. Only the difference in potentials, which is ultimately the gauge, constitutes a sensible measure. Because Maxwell's laws of electromagnetism remain the same under different segments of the potential scale, gauge theory concludes that likewise the laws of physics must remain invariant under gauge transformations thru the vacuum. This constraint however fails to capture the most significant gauge groups in nature, which are the following (Fig. 1):

- Quark scale

- Elementary particle scale

- Atom Scale

- Molecular Scale

- Lattice Scale

- Karyotic Scale

- Planetary or Astral Scale

- Solar System Scale

- Galactic Scale

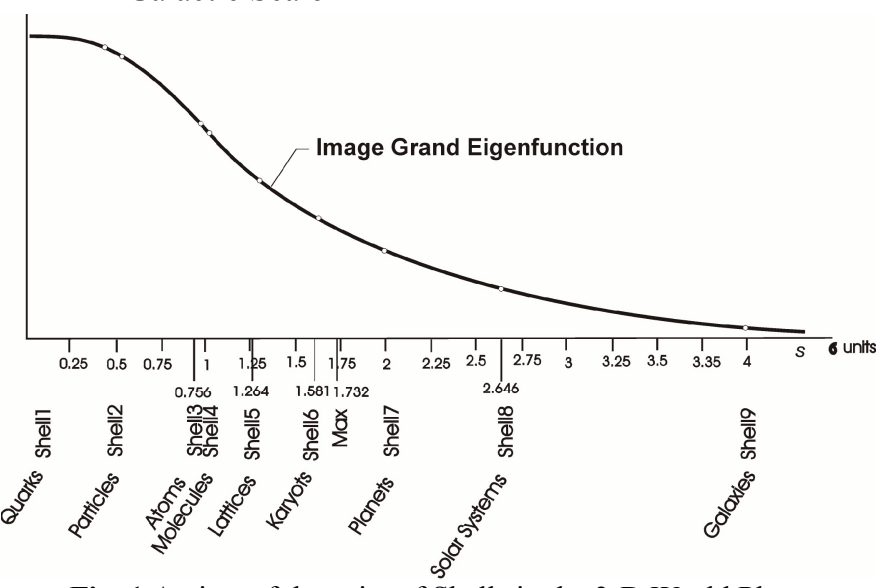

Fig. 1 A view of the suite of Shells in the 3-D World Plane
These scales and their topologies constitute what we call in Quanto-Geometry Shells of matter. It is incorrect to view the vacuum at each of these scales as absolutely equivalent to one another, even though there is an overarching character that is common to the vacua at all scales.

The real number line does not represent the physical scale for the 3-dimensional vacuum as a whole, viewed thru all Shells, that is. As we have stated in [1] and [3], the most towering metric for the continuum of the void is the $s$-metric whereby the elements or units of the scale stand for qualitative descriptors despite the fact that they are expressed in numeric quantities (Fig.2).

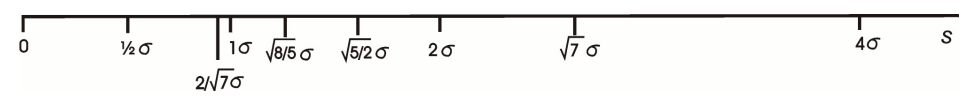

Fig. 2 Primeval sequence overscale of degrees of freedom laid on the $s$-axis

Notably, these quantities are accompanied by the $\sigma$ figure, which we shall discuss later. To venture a comparison with gauge theory, these numbers stand for the gauge potentials, but they are sensible in and of themselves even though it is the interval between two subsequent values that determines a Shell, or a Quanto-Geometric gauge group if you will. One of the reasons why these numbers bear physical meaning per se is because they represent the numeric quantities that give rise to the class of Quanto-Geometric tensors which specifically qualify the continuum of the vacuum in each Shell of matter [1] [3]. Whilst the most towering laws of physics will remain applicable in all Shells, each Shell is governed by its own foundational quantity or "fundamental physical constant", which in this case are nothing else altogether than the fundamental coupling constants. Most of these coupling constants we empirically know and peruse but have never recognized as such due to our ignorance of their origin.

We have shown in [1] how and why each Shell falls under the regency of a particular coupling constant as mathematically derived from first-order Quanto-Geometric principles or the Quanto-Geometric axiomatic.

\section{Incomplete Understanding of Nature's Symmetry Orders in Modern Physics}

The above naturally takes us to a brief discussion concerning the notion of symmetry in physics as universally regarded in all theories [16]. Our common definition of physical symmetry calls for an invariant observable under a larger frame of transformations, making a symmetry group of all the transformations under which that invariant is preserved. As important as it has been in our physical theories, this view 
of symmetry only belongs to a lower $3^{\text {rd }}$ or $4^{\text {th }}$ level of nature's symmetries, other higher-order symmetries being more instrumental in the determination of the fundamental quantities that ground the 3-dimensional edifice.

When we start out with the symmetry group being the set of transformations under which an observable is preserved, such as the translational or traversal groups of reference frames, we are automatically removing from the scope of the Theory the ontology of the observable and constraining the Theory to strictly be a phenomenological description of reality. In the theories of Relativity for instance, in so doing we put the brunt of the analysis on the coordinate reference frames which are unable to describe the action by the underlying vacuum and only amount to an overhead methodology to describe the optics and dynamics of light in the vacuum. Likewise in Quantum Field Theory, spin groups are unable to describe the details of the ultra-structure of the particle that creates the spin, to the point that many physicists equivocally take the spin simply as a metaphor and not a real physical effect that is explicit in particles' behavior.

What we have to recognize is that the void of space not only provides the medium for traversals in all regimes, whether constant multivariation or accelerated multivariation, but represents the very multivariate engine. The invariant observable only inherits a property attributable to the scalar which is ontologically static. All of which makes the Shell not only a phenomenological stack but truly a meta-level object.

All Shells are built on a fundamental invariant in a variance by a spatial spread that permits or mediates traversals. That invariant incarnates the fundamental physical constant. A fundamental physical constant is a unified quantum-space quantity that remains preserved under all actions, whether conversions, transformations or traversals, taking place topologically within a specific Shell of matter. In that sense the sustaining fundamental physical constant plays the role of intangible building block of the Shell.

The symmetry breaks at the "limiting" spread of each Shell to give rise to the next one up without a gap in the continuum. Therefore every wavenumber point on the scale (or the over-scale) of the continuum is a symmetry-breaking point beyond which the tapestry of the continuum becomes richer in spread, in degrees of freedom and in primal symmetries. Primal symmetries are the primitives to the order of symmetry [2]. This is the context in which all conservation laws must be understood, and it is the context that creates or promotes the conservation laws that we have become familiar with, in the understanding that there still exist elements of the Quanto-Geometric canon that lie beyond.
That all said, there is no doubt that the theories of Relativity are more assertive with the vacuum than the Quantum Theory has proven to be.

\section{The Vacuum thru the Planck Scale}

So much has been built on the basis of the Planck scale that many today seem to forget or misunderstand that it is only a hypothesis [8], but not a real physical scale. The Planck scale is only our modern physics view of how the infinitesimal should behave. There is indeed no Planck scale in the quantum physics of nature to be observed. If the putative Planck scale cannot seamlessly connect to the cosmological scale at the first place, then it can only remain an artificial useless scale, just because the continuum from the microscopic world of quanta to large-scale structures in the cosmos is an ostensible and inarguable observable.

This scale had been visualized by Max Planck himself without assigning any quantity to it [8]. In time, physicists have managed to correlate the speed of light as a fundamental quantity characterizing the quantum of light to other physical variables in order to project their possible minimal quantities or expression. Evidently this effort is developed on the wrong track since the first requirement for the development of a quantum scale is that of a quantum metric, which must duly incorporate the quantum vacuum as an essential element. Since most metrics if not all metrics that have been devised for the cosmological such as the FLRW ${ }^{1}$ metric incorporate a time coordinate, it is presumably difficult to likewise incorporate such a coordinate variable in a quantum metric, given that the quantum realm is the realm of the time-less par excellence. That is probably why you find some theoreticians with the insistent desire to pervert the Heisenberg uncertainty principle into:

$$
\Delta E \cdot \Delta t=\hbar
$$

so reflecting their inability to conceive the physical world without time. Consequently, and to our best knowledge, a quantum metric has not been formulated, hence the continued use of the Planck scale notion in modern physics. As far as our present interest in a characterization of sorts of the vacuum is concerned, one might wonder whether the Planck length specifically is a usable or suitable quantity at all to that aim.

From the perspective that we have maintained in modern physics thus far, the speed of light $c$ appears to be the most

\footnotetext{
${ }^{1}$ The Friedmann-Lemaître-Robertson-Walker metric
} 
fundamental of all constants of nature, in fact we should state the invariant motion of light rather. When we grade that motion in $\mathrm{m} / \mathrm{s}$ as a speed, we are positing that time must exist and that there must be a unit of time in nature. Thus far however, a natural unit of time has not been found, and instead we have found that we must deconstruct time, by inflating its basis unit in what Relativists have called time dilation, in order for $c$ to hold as an invariant quantity as observed. Therefore the inexistence of a universal unit of time implies not that $c$ is unreal as an invariant, but that the $\mathrm{m} / \mathrm{s}$ scale is unreal. Since the meter or the distance metric is an undeniable observable, we must hold that $c$, whatever its descriptive numeric quantity, is strictly a value whose meaning is exclusively rooted in the distance metric. At a minimum $c$ must become the unit for invariance, in other words a primitive etalon, as much as we must conclude that motion is rooted in the very distance metric exclusively. The latter means that the length between two space points, any set of two points, is not only the measure of a distance but very compellingly the measure of a quantity of motion as well, as we have stipulated in the development of the quantogeometric metric [1] [3]. This scale is fittingly the $s$-scale of quanto-geometric coordinate space, a wavelength or wavenumber scale.

Consequently the deconstruction of time undertaken in Relativity and its unredeemable defunction in Quantum Mechanics lead to a defection of the notion of a Planck length, because the relation:

$$
c=\frac{L_{P}(\text { Planck_length })}{t_{P}(\text { Planck_time })},
$$

the fundamental relation sustaining the notion of a Planck scale, cannot be upheld. Therefore we must abandon the idea of parcels of space because space as a continuum is totalitarian, boundless and indivisible in spread, to only visualize differentiation in space exclusively as a tensilefoliate order, origin of all forms of variance or multivariation.

We will further assert that the only useful and real figure with regards to Planck discretisation is the Planck energy density unit $h$, the Planck constant, which is only applicable to particle objects, but not the basis coordinate space in the 3-D arena. So it is because it is not $h$ that creates the primitive state of the vacuum but the primitives associated with the vacuum that give rise to $h$ just as one element in a class of primordial Shell constants.

\section{Why so Many Different Ways to Compute the Vacuum Energy Density in Quantum Field Theory?}

Incredibly enough, there are at least 4 different methods in Quantum Field Theory to compute the value of the vacuum energy density, each casting a different quantity and none of which being in agreement with experimental observation. In the particular theoretical method being used, calculations of $\rho_{\text {hep }}$ may depend on: identification of relevant contributions of different particles/fields, choice of best-fit ultraviolet cutoff, possibility of various cancellation mechanisms, etc. According to F. Wilczek (2001) one may estimate a $\rho_{\text {hep }}$ of about $10^{108} \mathrm{ev}^{4}$ based on the standard quantum gravity/Planck scale cutoff method, a $\rho_{\text {hep }}$ of about $10^{96} \mathrm{ev}^{4}$ based on unified gauge symmetry-breaking, and a $\rho_{\text {hep }}$ as small as $10^{44} \mathrm{ev}^{4}$ if "lowenergy super-symmetry enforces, as he put it, big cancellations." In summary, $\rho_{\text {hep }}$ values can range anywhere between $10^{92} \mathrm{~g} / \mathrm{cm}^{3}$ and $10^{30} \mathrm{~g} / \mathrm{cm}^{3}$ approximately.

Why such wildly differing numbers inside the Theory and why such a big discrepancy with the observed or experimental value? No solid theory with the ambition of harnessing the vacuum can afford predictive mishaps this magnitude. Since every one of the methods employed for the computation is germane to fundamental aspects of the theory, and since everyone seems to acknowledge that these numbers are altogether wildly inaccurate, one has to conclude that the theory's assumptions and postulates are wrong and refrain from attempting to cure these numbers from inside the Theory. One can either choose to stick to the Theory and ignore its vacuum crisis or engage an entire rebuild of the Theory in a way to preserve its successes while ensuring a path consistent with first principles toward assessing the vacuum energy density assertively and straightforwardly. What one should not attempt to do is to create artificial means inside of the Theory to come up with a more or less acceptable number [6], because this would be equivalent to studying a subject just to pass the assessment tests, remaining largely ignorant of the fundamentals of the subject. On the other hand, one may obviously choose to approach the question of the ontology of the vacuum from an entirely different perspective from QFT, as we have.

To the end, the answer to the question posed is that the Theory has no clue or no good clue.

\section{Quanto-Geometric Mathematical Characterization of the Vacuum}

In [1] and [3] we have put on the table a timeless metric description of the Universe, dubbed an Omniverse, that 
initially casts 4 numbers which work and walk tightly together in functional analysis dominated by an Eigenfunction, as shown below (2). The details of the analysis are undertaken in [1], and reported in [3] in some measure as well.

$$
\begin{aligned}
& n=D_{3}=3 \\
& s_{3}^{-1}=1.322875656 \sigma^{-1} \\
& q_{3}=0.299796065 \sigma^{-1} \\
& \sigma^{3}=1.00003609 \cdot 10^{-27}
\end{aligned}
$$

$\boldsymbol{n}$ being the variable in a sequence $\left\{\mathrm{s}_{\mathrm{n}}\right\}$ that determines the dimensional modulus $D$ for each World Shell or World Plane of the Omniverse, a structure that could be heuristically compared to an onion ball.

$\boldsymbol{s}_{3}$ being the variable expressing the spatial wavefunction of the 3-dimensional World Shell of the Universe, somewhat similar to the visualization of the 3-D Universe's wavefunction $(|\psi\rangle)$ underpinning the Wheeler-Dewitt basis equation for the 3-D universe [7], as reported below:

$$
\hat{\mathrm{H}}(x)|\psi\rangle=0 \text {. }
$$

$\boldsymbol{q}_{3}$ being a scalar density figure which determines the propagation moment of space in the 3-dimensional World Plane.

$\boldsymbol{\sigma}^{3}$, energy density of the vacuum with $\boldsymbol{\sigma}$ being originally the standard deviation of the Omniverse.

These numbers stand for pure mathematical scalars, and constitute a direct response to the call of David Hilbert's Sixth Problem for axiomatization of Physics [12]. In assessing the validity of these numbers and their possible contribution to the resolution of the vacuum crisis, it is worth analyzing them in the light of D. Hilbert's motivation for mathematical axiomatization of Physics. The latter is an equivalent view to the long-sought theoretical derivation of the fundamental physical constants in Physics [20].

In passing, we shall highlight the very important fact that the vacuum crisis is a clear reflection of the deep incompatibility between the Theories of Relativity and the Quantum Theory, which amounts to a severe defeat in the work of unification of both Theories, considering that the most important impulse for the development of Quantum Field Theories was the goal of unification.
The numbers above cited are physically unit-less, except for $\sigma^{3}$. They cannot have any physical unit-dimensions because they are pure mathematical engenders, to the same extent as $\pi$ or $e$. This result should be expected if the theoretical derivation of the fundamental physical constants must strictly come through pure theory or mathematical axiomatization. Therefore one should never expect the known dimensional quantities such as $c$ to be directly cast by the mathematical axiomatic. One may well counter argue that the dimension-less fundamental constants such as $\alpha$, the fine structure constant, should be cast as is by the axiomatic system. While this is a consonant expectation, we should not lose sight of the important fact that the physical constants are not independent from one another, that they exist in a hierarchical scaffold of dependencies, and that what appears to be dimension-less or unit-less within our unit-based system could have a totally different figuration in a purely mathematical system, despite being non-dimensional.

\section{Expectation Methods in Theoretical Derivation of the Constants of Nature}

\section{Number magnitude}

In evaluating the fundamental quantities, the next question to consider is that of number magnitude. Nothing in physics allows us to judge or gauge a number magnitude in and of itself. The question of number magnitude is a purely mathematical question, the magnitude being given leaving no other choice to Physics than to accept the figure, just as the science had chosen to completely rely on mathematics and its methods for its development and analytic results. The fierce imperative of mathematical dictates is sovereign in the question of magnitude of derived numbers from the axiomatic.

\section{Number figuration}

Does an axiomatically derived number gain credit just by carrying an identical suite of digits from the unit position on down to the known physical figure? From the perspective of our Number Theory in its current state in mathematics, the straight answer is no without a doubt. Simply because the exact same figuration for one particular case might just happen to be fortuitous.

However when a whole series of quantities happen to show a figuration match as defined above throughout the hierarchical scaffolding of numbers, one is no longer facing a fact of serendipity but a trend. That trend duly validates the legitimacy of the specific suite of numbers in the quantity. 


\section{Translation}

The first level of translation from the numeric scaffold to the physical or dimensional quantities follows from the above consideration relating to figurate match. Wherever a figurate match is acknowledged then the entire derived number must be considered at the very least a precursor to the known physical quantity.

Not knowing what form a purely derived quantity might represent, the question of translating the theoretically derived fundamental constants to the empirically known values had been raised. Evidently the elements of the axiomatic have to hereby play an important role since it is only a match between contextual principles surrounding the matching quantities in both systems that can provide a basis for comparison of the relevant quantities.

However once an axiomatically derived number has achieved the precursor status, that situation trumps the need for comparative contextual representation for a match to be acknowledged. The precursory number then encapsulates the most important definition and expression of the principle in question within the entire mathematical-physics system.

\section{From Mathematics to Physics: is there a Common Node or a Translation code?}

This question means to simply ask whether or not there is a preferred unit-dimension to be matched with any possible figure within the mathematical axiomatic results in order to achieve full physical translation of the mathematical quantities scaffold.

This question for sure invites another, which is: what happens when an arbitrary unit system is not in resonance with the elements of nature in the universal arena? One answer to the question seems to be that in that event one will tend to view in nature a lot of polemic facts or events, a.k.a. our so called "anomalies" of nature which are many, just because the unit system ported by the instrumentation and the very nature of the instrumentation cannot view reality in its native structure or ultra-structure. Should we let alone the question of what happens when one uses a fundamental unit at the top of a unit system that has no replicate in nature? Am I alluding to time? Sure I am. To make this clear, if our sense of sight i.e. was not in tune with the invariance of the speed of light $c$ thru air, transparent materials or vacuum throughout the 3-D arena, in all observational directions simultaneously and for all inertial observers, we would not be able to see any object in its very native structure and appearance, everything would appear to us instead like mirages or complete or partial aberrations of themselves. Instead our sense of sight brings us the world exactly as is, and that is what being in tune with nature means.

So therefore our unit system appears to be as arbitrary as anything can ever be. Can there be a norm or a criterion to select one particular empirical physical unit or quantity for a parameter match to the axiomatic system, so that the derived fundamental scalars can become physical quantities? My answer to this question is that the results from this application will not necessarily be widely consistent across the entire scaffold, not because of inevitable errors or inaccuracies in experimental measurements, but due to improprieties within the unit system itself. So therefore it is my sense and hope that the fundamental quantities derived from mathematical axiomatization of physics will help deeply cure the unit system, which has historically been a matter of troublesome if not traumatic continual revision.

\section{Parameter Match for the Quanto- Geometrically Derived Fundamental Constants}

The Standard Bodies have recently taken the decision to promote the meter with a change in status from a simple unitdimension for distance to a full-fledged physical constant [19]. This motion is part of the strategy lately adopted by the standard bureaus to reduce the dependency of the standards on guarded etalons in continually passivated environments, an overly difficult undertaking.

This motion, however, while useful in helping eliminate an etalon, does not address the establishment of a metric scale that is applicable not only for the wider world of our habitat, but for the infinitesimal quantum world as well. Meanwhile in the physical science of spectroscopy as well as optical engineering, physicists and engineers respectively have long been using the sub-region of the metric scale, in the personification of the nanometer unit, in order to quantify wavelengths of light throughout the radiation spectrum. Given that situation, one may rightfully consider the nanometer to inherit the qualities attributed to the meter as a unit and a constant.

It is a remarkable fact that the standard bodies have officially defined the meter as the distance travelled by light of a specific frequency for the duration of 1 second, while the radiation itself is already characterized by a distance figure, which is its wavelength. Even though the NIST takes express care to refer to the frequency of the elected radiation line in the definition, it remains nevertheless true that the wavelength is the primary descriptor of light radiation and in measurement practices stands for the most frequent and suitable 
characterization in use. If there is something directly measurable in the harmonic behavior of light, it is its wavelength or its wavenumber (reciprocal of wavelength) thru diffraction, dispersion and interference patterns, and not its frequency, since nobody has ever seen under an oscilloscope or an optical spectrum analyzer an eternal sinusoid that is directly brought up from a light beam but only a simulation of the same thru photodiode arrays. There is certainly a discussion to be had about the fact that light, visible and invisible, $x$-rays and gamma rays considered part of the electromagnetic spectrum, are directly radiated by atoms where there is in existence no time-driven phenomenology, while the other electromagnetic waves of the spectrum have to be generated thru an artificial antenna rod or cavity. We extensively carry out this discussion in Chapter 8 of [2].

We should further note that the wavelength and frequency figure for light does not quite have the same definition as they do for other periodic phenomena based on the eternal sinusoid. For those other type of harmonic phenomena, the frequency is the number of cycles per second, and the period or wavelength is the inverse of the frequency $(1 / f)$, measured in time unit, and defined as the time it takes a complete harmonic cycle to complete itself [17] [18]. Whereas for light, the wavelength is measured in unit of space or length, the nanometer, and defined as the distance over which the propagating wave completes a harmonic cycle. The frequency is determined from the wavelength thru the formula:

$$
f=\frac{c}{\lambda}
$$

Consequently, even though the official definition of the meter seems to be going around in a vicious circle, the life of the meter standard has been continually revolving around measurement of harmonic phenomena, not just topography. And we have to stress that in its intervention in the harmonic world with the characterization of light specifically, the meter standard does so on the basis of treating light as a space-driven phenomenon thru the wavelength or wavenumber figure and not as a time-driven phenomenon. For these reasons, we have chosen to make a match between $\sigma$, our putative mathematical unit, and the physical nanometer both in its condition as a unit-dimension and a constant. Most importantly, we have elected to make this match because in characterizing length the nanometer also characterizes a wave-length. As a reminder, in the Quanto-Geometric metric [1] [3], the $\sigma$ parameter is a qualifier for both length (topographic length) and wavelength.

\section{From Momentum Space to Space Momentum within the Fabric of True Coordinate Space}

So therefore we have chosen to match the standard deviation parameter $\sigma$ with the physical unit/constant of the nanometer, so that:

$$
\sigma=1 . \overline{0} \cdot 10^{-9} \mathrm{~m}
$$

We have further decided to adjust that quantity in its $10^{\text {th }}$ decimal place, in order to obtain an exact 9-digit match, instead of a 6-digit match at first order, with the quantity known as the magnitude of the speed of light, thru the $3^{\text {rd }}$ relation in (2), as explained in [3]:

$$
\sigma=1.00001203 \cdot 10^{-9} \mathrm{~m}
$$

As reported in [1] and [3]:

$$
q_{3}=\frac{0.299796065}{1.00001203} \times 10^{9}=299,792,458 \rightarrow \mathrm{m} / \mathrm{s}
$$

The second relation in (2) puts before us another important quantity characterizing the ontology of space in the 3dimensional World Plane:

$$
\begin{aligned}
& s_{3}^{-1}=1.322875656 \cdot \sigma^{-1} \text { (6) or } \\
& s_{3}=0.7559289458 \cdot \sigma \quad \text { (7) }
\end{aligned}
$$

The quantity in (6) expresses the wavenumber of space in the 3-dimensional World Plane and represents a clear precursor of the Compton Wavelength of the proton, whilst the quantity in (7) expresses the wavelength of space in the 3-D World Plane and represents a precursor to the atomic mass unit-inverse meter relationship.

By replacing $\sigma$ in (6) and (7) with the adjusted value in (5) we obtain:

$$
\begin{aligned}
& s_{3}^{-1}=1.322875656 \cdot 10^{9} \mathrm{~m}^{-1} \quad \text { (8) and } \\
& s_{3}=0.75593804 \cdot 10^{-9} \mathrm{~m}
\end{aligned}
$$

The proton Compton Wavelength is known to be equal to:

$$
1.321409856 \cdot 10^{-15} m
$$

The atomic mass unit-inverse meter relationship is known to be equal to: 


\section{$0.751300661 \cdot 10^{15} \mathrm{~m}^{-1}$}

Without entering into the defining details of the atomic mass unit, undertaken in [2], let's briefly note that the atomic unit of mass is conceptually extremely close to that of the proton as well as that of the neutron individually, given that: 1) these two particles almost have the same mass value and 2) the mass of the electron is totally negligible in the context of atomic mass and the averaging of the mass of atomic protons and neutrons for a mass unit for atoms. Therefore the so-called atomic mass unit-inverse meter relationship tightly relates to the proton, and could well be construed as the "proton massinverse meter relationship". The obtuse dimensional meaning of the cryptic nomenclature officially assigned to this quantity should not deter us from:

- relating it to the proton Compton wavelength

- noticing that its magnitude expressed in $15^{\text {th }}$ power of 10 correlates by conjugation to that of the proton Compton wavelength $\left(10^{-15}\right)$

- noticing that its dimension $\left(m^{-1}\right)$ is exactly the reciprocal of that of the proton Compton wavelength $\left(m\right.$ or $\left.\mathrm{m}^{l}\right)$, which makes it interchangeable with a wavenumber

- heeding the paramount fact that these two quantities are reciprocal to one another, not only in their physical unit-dimensions, but very much numerically as well, at least in their 3 most significant digits.

Note that these results would still hold in their entirety even with the original non-adjusted value of the nanometer for the physical translation of $\boldsymbol{\sigma}$. Also note that the context in which the atomic mass unit-inverse meter relationship is obtained as $(\mathbf{l u}) \boldsymbol{c} / \boldsymbol{h}$ is completely independent from that of the proton Compton wavelength at $\mathrm{NIST}^{2}$, an important fact that cannot be overstated. Yet in their three most significant digits, these two quantities (proton Compton wavelength and atomic massunit inverse meter relationship) are reciprocal to one another.

All these traits respectively apply to (8) and (9), and add to the figurative match between the two sets of quantities. While there may be further transformations to be applied to (8) and (9) in order to arrive at the two cited empirically known quantities, the role of $s_{3}$ and its reciprocal $1 / s_{3}$ as highlevel precursors in their generation is undeniable.

The above analysis compels us to draw the unexpected yet outstanding conclusion that the notion of momentum space

${ }^{2}$ NIST is the United States' National Institute of Standards and Technology. in QFT, modeled after Hilbert functional spaces is far from an abstraction but a real characteristic of coordinate space. At the same time, this realization pulls the rug under the towering relation upon which QFT is built [14], which is

$$
p=\hbar k
$$

where $\boldsymbol{k}$ is the wavevector, $\boldsymbol{p}$ the momentum and $\boldsymbol{h}$ the reduced Planck constant. Momentum space or reciprocal space is matter-of-factly defined, in the Quanto-Geometric framework, at a much higher level than $h$, the Planck constant, within the hierarchy of symmetries upon which the 3-D World Plane is built. The cluster of fundamental quantities in (2) represents the foundational pillars sustaining the 3-D World Plane. Under the Quanto-Geometric vision, true reciprocal space is spelled out in (8) whereas (9) describes more closely what QFT would consider to be reciprocal space. In the end, it matters not what approach we take to the description of momentum space, whether the straightforward view of $s_{3}$ or its reciprocal $s_{3}^{-1}$, because they both directly relate to the $3^{\text {rd }}$ relation in (2) which describes the propagation moment of $s_{3}$ space, a real quantity typifying $c$ motion, indeed the first of all motion in 3-D World Plane. True coordinate space is endowed with a wavelength or a wavenumber materializing what the Quantum Theory has designated the wavefunction, as well as a propagation moment or a deployment speed. Indeed the notion of space momentum (in lieu of momentum space) encapsulates the best visualization of the seminal activity of the physical vacuum which creates multivariation or motion, as analyzed in Chapter 7 of [1].

What the QFT towering relation (10) is attempting to capture is the defining relation of momenta, which is the Abelian association between a scalar tenet, in this case energy density, and the velocity of the moving object or energy packet, in this case portrayed in a vector or wavevector. This representation is epitomized in Quanto-Geometry by the Grand Eigenfunction where $Q(s)$ is the scalar density and $s$ is the independent dynamic variable or spatial wavelength. When, under the mandate of an index of symmetry $n_{3}(n=3$ or $n_{3}$ ), the variable takes a certain value $s_{3}$, then $Q(s)$ results in a certain value $q_{3}$, so that we obtain the pilot cluster of quantities (2) cementing the foundations of the 3-D arena. Of the essence is it to comprehend the fact that the precursory term to what we call the speed of light is not at all a direct characterization for light which is a lower level phenomenon in the buildout of the 3-D World Plane. This quantity of motion (or Lorentz invariance) is to be tributed to coordinate space and describes its innate activity in the form of propagation, deployment or traversal. The $c$ motion is the motion of coordinate momentum space, an intrinsic spatial 
momentum, and stands for the first of all motion in the 3-D arena.

Perhaps a metaphor may help visualize the notion of physical space momentum in relation to the quantum of light. Let us presume that a surfer, mounted on a surf board, are both in a cloth coating that polarizes light in one direction. Let us further presume that the ocean water bears a surface coating that polarizes light in an orthogonal direction to the first. Lastly let's visualize an observer on the beach wearing polarized glasses in the same direction as that of the surfer and his/her surfboard. What the observer will see is a vast spread of darkness in the midst of which a surfer is moving in all kinds of directions at will. If this observer happens to have never seen the ocean before, he/she will genuinely believe that the motion of the surfer throughout this vast spread of darkness is his or her own and not in fact the motion of the sea waves to which the surfboard is hooked. The surfer is comparable to the quantum of light, and the ocean with its multitude of waves is comparable to coordinate space with its wave-function property.

Because we are used to wrongly associate motion natively with mass, through the classical physics of impacts and forces, it is difficult for us to visualize motion without mass involvement. However, space not only provides the medium for motion but it also provides the impetus of motion, which A. Einstein had incipiently visualized as geodesic guides within the geometry of space [9]. Per Victor de Broglie wave theory, a wavefunction is associated with every object, and this wavefunction is pure spatial motion, it is not an abstract function or functional. Quanto-Geometric Theory teaches that this wavefunction ought to be visualized as a wavelength innate to spatial vacuum, that this wavelength is an independent variable to another Function which relates that wavelength or harmonic phenomenon to a scalar density packet operating in covariance with the wave tenet.

The numbers that accompany this vision, because they are reflected in the empirically known foremost fundamental physical constants in one capacity or another, prove this vision peremptorily correct. Note that this characterization applies to the vacuum in the 3-D World Plane as the totalitarian background vacuum. The vacuum in the derivate Shells of the 3-D World Plane inherit that ubiquitous background trinary tensor [3] which is superimposed on its breaking into the local symmetries fashioning each Shell (Fig. 1).

\section{Relativistic Vacuum Energy Density}

By studying orbits of particles moving in the gravitational field of known masses, cosmologists had been able to arrive at consistent and reliable values of the vacuum energy density in the 3-dimensional Universe [10] [11]. The larger the considered system the more accurate the estimation, since we are looking for a constant density.

\section{Estimating the value of the Vacuum Energy Density in Cosmology}

Given that the Solar System is the largest system where we hold full knowledge of bodies' mass quantities, we can start by checking for the presence of a vacuum energy density via a careful test of Kepler's Third Law, one according to which the square of the orbital period is proportional to the cube of the distance from the Sun.

The centripetal acceleration of a particle moving around a circle of radius $R$ with period $P$ is

$$
a=R\left(\frac{2 \pi}{P}\right)^{2}
$$

where $a$ is the centripetal acceleration, $R$ the orbiting radius and $P$ the period of the orbiting motion. The centripetal acceleration of the particle ought to be equal to the gravitational acceleration, according to:

$$
a=R\left(\frac{2 \pi}{P}\right)^{2}=g=\frac{G M_{S U N}}{R^{2}}-\frac{8 \pi}{3} G \rho_{V} R
$$

where $G$ is the Newton gravitational constant, $M_{S U N}$ is the mass of the sun star, $\rho_{V}$ is the vacuum energy density inside of the orbiting radius. If the energy density of the vacuum was to be equal to $0\left(\rho_{V}=0\right)$, we would obtain Kepler's third law from the above:

$$
P^{2}=\frac{4 \pi^{2}}{G M_{S U N}} \cdot R^{3}
$$

If the energy density of the vacuum is held to be non-zero instead, then the period changes by a small fraction, according to:

$$
\frac{d P}{P}=\frac{4 \pi}{3} \cdot \frac{R^{3} \rho_{V}}{M_{S U N}}=\frac{\rho_{V}}{\bar{\rho}},
$$

which sets the average vacuum density inside of the radius $R$ to be equal to:

$$
\bar{\rho}=\frac{3}{4 \pi} \frac{M_{S U N}}{R^{3}}
$$


Our modern spacecrafts that are able to determine very precise distances to such planets as Neptune and the other large planets have found a variation for $d P / P$ to $1+/-1$ part in one million at Neptune's distance from the Sun. From such observations, a limiting value to the vacuum energy density within the solar system may be estimated at:

$$
\rho_{V}=5+/-5 \cdot 10^{-18}<2 \cdot 10^{-17} \mathrm{~g} / \mathrm{cm}^{3}
$$

The next approximation level for the value of the vacuum energy density is to consider the Sun's orbit around our Milky Way galaxy and make an estimation for the vacuum inside of that orbit. This estimate casts an approximate value of:

$$
\rho_{V}<\frac{3}{4 \pi G} \cdot\left(\frac{v}{R}\right)^{2}=3 \cdot 20^{-24} \mathrm{~g} / \mathrm{cm}^{3}
$$

This estimation takes into account a circular orbital velocity of $200 \mathrm{Km} / \mathrm{s}$ and a distance $R=8.5 \mathrm{kpc}$.

The next and last best approximation level for the value of the vacuum energy density is to consider the Universe itself as a whole. We can no longer consider orbits in this case but the observed accelerating expansion of the Universe. Considerations about the parameters of this expansion have set the limiting value for the vacuum energy density around:

$$
\rho_{V}=0.8 \cdot 10^{-29} \mathrm{~g} / \mathrm{cm}^{3}
$$

To be even more precise, cosmologists have computed based on these approximations a critical value of the vacuum energy density that is pretty close to the above quantity and which determines the evolutionary expansion of the Universe. We note that the supernova data gathered by the Voyager and other probes do lend credence to the above computed value since they amount to a critical $\rho$ value of $0.6 \times 10^{-29} \mathrm{~g} / \mathrm{c}^{3}$. We will come back later to the critical value of the vacuum energy density.

It is important to highlight that the measurement unit of $\mathrm{g} / \mathrm{cm}^{3}$ for the vacuum energy density in the analysis does not directly characterize the vacuum since there is nothing inside of the vacuum to weigh in $g$ or $\mathrm{kg}$. What we have done in cosmological science is to establish equivalence with matter density on the basis of relativistic matter-energy equivalence. Note as well the very important fact that at each Shell of matter, the estimated vacuum energy density is different, the density being lower for larger volumes of the vacuum.

\section{Closing the Gap between the Quantum Vacuum and the Cosmological Vacuum}

By contrast, in Quanto-Geometry, we hold a direct measurement qualifier for the vacuum, which is the mathematical unit of $\sigma$, the standard deviation of the Grand Eigenfunction. Sigma $(\sigma)$ measures the variance of $s$-space as an independent wavelength variable to the function and constitutes the unit of the Quanto-Geometric super-metric. Therefore, in its application to the 3 dimensions or degrees of freedom of space in the 3-D World Plane, we have to elevate $\sigma$ to the cube in order to obtain its expression for the 3-D World Plane. Keep in mind that every World Plane develops by emulation to the entire Omniverse, which stands under the direct regency of the Grand Eigenfunction at first order. Having matched $\sigma$ to the nanometer constant for a physicalworld incarnation in 3-D World Plane as shown in (5), we obtain:

$$
\begin{aligned}
\sigma^{3} & =\left(1.00001203 \cdot 10^{-9}\right)^{3} m_{E}^{3}, \\
\sigma^{3} & =1.00003609 \cdot 10^{-27} m_{E}^{3}
\end{aligned}
$$

where the unit $m_{E}^{3}$ stands for cubic meters of harmonic energy "fluctuations". Whether or not one wants to make the quantity the value of "energy fluctuations" per cubic meter of the vacuum is entirely left to their preference. If we set aside the somewhat debatable question of legitimacy of the unit figure of $m_{E}^{3}$, we readily notice the closeness in magnitude of this number to the cosmological vacuum energy density, contributing at once to eminently close the gap between the quantum vision of the vacuum and the relativist cosmological vision, as well as the disparity in their individual quantification of the same. We only notice a difference of just 2 orders of magnitude, which compensates the declared and acknowledged approximations in several instances within the cosmological calculation methodology. For that matter, we give complete primacy to the theoretically derived quantity over the empirically computed quantity. Because $\sigma$ is a highlevel parameter of the Omniverse that applies to the entire continuum of 3-D Shells as one point, $\sigma^{3}$ consequently applies to all Shells as well [1] [2]. In that condition it has the same weight as the fundamental parabolic tensor that subtends all of 3-D space and acts as a grandfather to all other tensors that typify the many different 3-D Shells, including any circumstantial or regional tensor, in the vicinity or not of large masses, as generally known in the relativistic treatment.

I will not conclude this section without stating that, if given a choice, we would rather not characterize content of the 
vacuum in terms of energy density. This is because the notion of energy is not a first-order characteristic of the vacuum, since within the Quanto-Geometric canon we have derived the energy constants at a lower order in the scaffold, both the thermodynamic unit of energy in the embodiment of the Stephan-Boltzmann constant and the quantum unit of energy embodied by the Planck constant. Consequently we would prefer in the above characterization the nomenclature of $m_{W}^{3}$ for the measurement unit, where $W$ stands for wavelength in substitution of $E$ for energy. It is important to understand and acknowledge that at its native or ontological level, the content of the vacuum is not one of Energy nature, if we accept the two mentioned constants as the heart of the two scales of energy in existence. This view certainly grants a breath of support to the initial harmonic conceptualization of the vacuum in QFT which led to the $\lambda_{C}^{3}$ unit, a dimension we acknowledge to be very similar to $m_{W}^{3}$. However, the erroneous application of the concept to points of the vacuum which led to the deadly Renormalization procedure irretrievably killed the initial insight.

\section{The Universe is not a Time Domain but a Stochastic Domain}

The above analysis drives us to the inevitable, but to many surely flabbergasting and shocking, conclusion that the Universe as a whole is not a time domain but a stochastic domain. The Omniverse is yes a stochastic domain, the World Planes are stochastic sub-domains. If the Quanto-Geometric Grand Eigenfunction is an ever faithful descriptor of the same, then this conclusion is an irrepressible one. Rejecting this conclusion is to forever buck at the cluster of fundamental physical quantities presented at the beginning of this study (2) and naturally obtained in functional analysis centered on the Grand Eigenfunction.

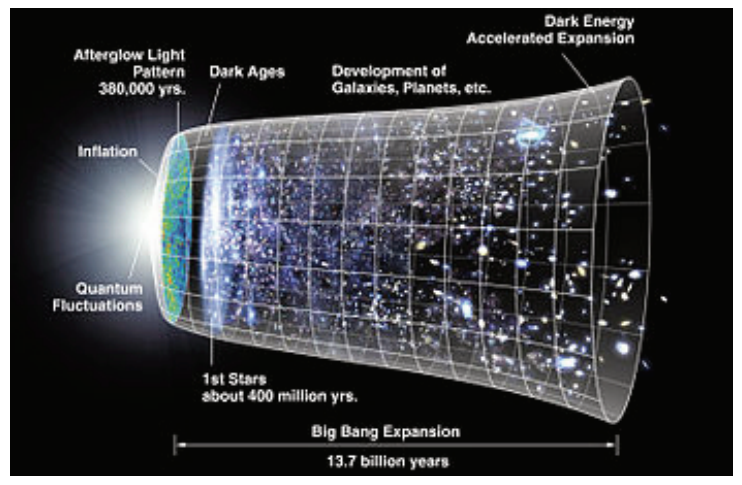

Fig. 3 Depiction of the narrative of Big Bang birth and time evolution of the Universe
The Standard Model of particle physics describes the history of the Universe along a timeline, consistent with its vision of the vacuum. The vacuum crisis is undoubtedly an indictment of Quantum Field Theory in its entirety along with its Standard Model. It is likely, in our reserved opinion, that only the politics of physics is preventing this realization to come to the fore. Further, we are just nowadays witnessing the plummeting of the most promising extension of the Standard Model in the embodiment of Supersymmetry. I hold the notion of time to be the most important culprit that has defeated Quantum Field Theory from its inception with P. M. Dirac, R. Feynman and other pioneers to date, a subject that I extensively treat in [2]. That is what makes this realization from F. Wilzeck almost visionary: "[The solution to the vacuum problem] might require, he writes, inventing entirely new ideas, and abandoning old ones we thought to be wellestablished."

The illustration in Fig. 3, ever so present or invoked in every text and textbook of advanced physics in every form, depicts the narrative of the history of the Universe that everyone else in mainstream culture has come to know as the Big Bang Theory. The universe has exploded into existence 13 15 billion years ago, and since then maintains an incessant expansion along an endless timeline, the story goes.

However, because there was an early inflation epoch, the hypothesis continues, a complete narrative is best represented in this other illustration below reported, less popular but more accurate (Fig. 4).

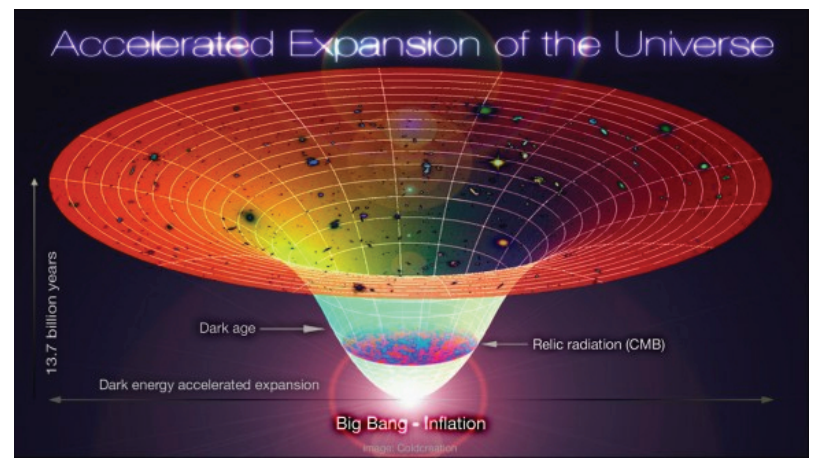

Fig. 4 A more accurate graphical depiction of time evolution of the Universe - Art by Wikimedia Commons

What is wrong with this picture? What is wrong with this picture is what is wrong with $\rho_{\text {hep }}$. This sort of visualization of the 3-D Universe and its history is in itself a contradiction. If this is how the Universe evolves in time, then the heart of the universe must be covariance between mass and space, which are everywhere the only observables, in a form of distribution that is equivalent to the familiar Gaussian distribution. Then 
that automatically takes time out of the equation and makes the distribution parameter of $\sigma$ an important descriptor of the vacuum in terms of its variance. So therefore $\rho_{\text {hep }}$ must be a $\sigma$ parameter and the time axis must be instead a scalar-mass density axis, evolving with origin set at the asymptotic plane. Effectively we do see in the 3-D World Plane the suite of Shells of matter with its higher level of mass density in the infinitesimal realm, reason why we call quantum physics highenergy physics, with the mass density gradually diminishing from Shell to Shell on up. The mass density orderly follows a diminishing trend until the outer (or top) Shells (galaxies and galaxy groups), whereas we witness the spread of spatial void evolving in an inversely proportional manner from Shell to Shell on up. Effectively, it is all not about mass but mass density and, further, the void of space is active: you have to pay attention and give value to the spread of the vacuum! In other words, the continuum of space along with its associated scalar-masses, from the quantum scale to the cosmological scale, is a density function with space vacuum as the independent variable (Fig. 4b).

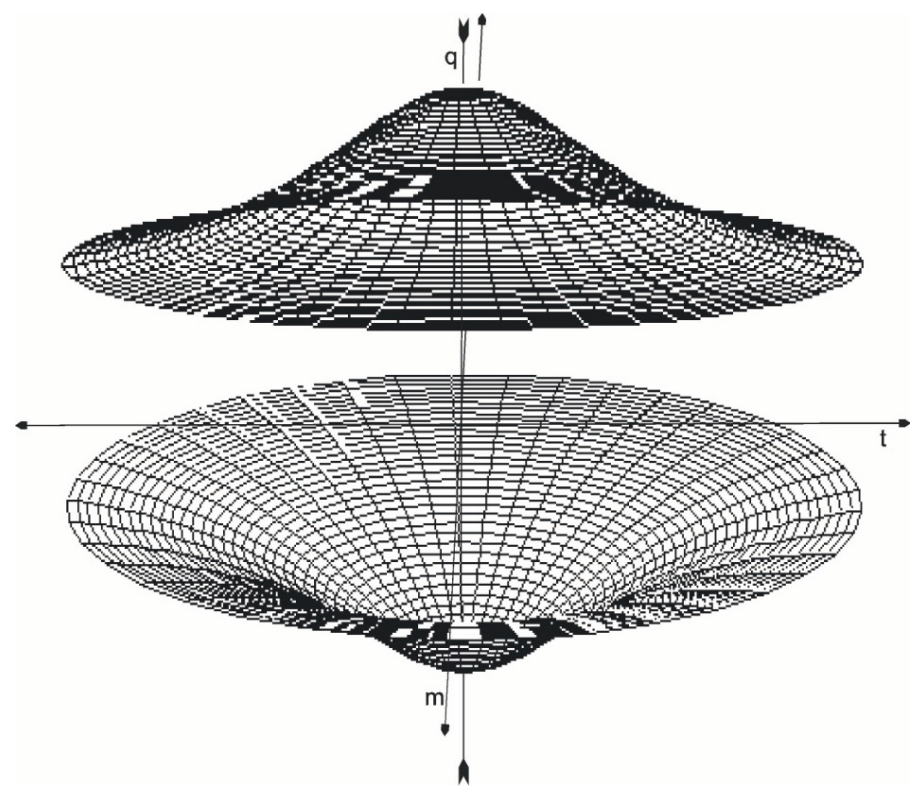

Fig. 4b A complete view of the graph of the Quanto-Geometric Eigenfunction, a function of two space variables $(m, t)$

Since the Gaussian function is a derivate of the QuantoGeometric Eigenfunction, the latter meaning a root function, the true real distribution of the observables in the cosmological arena represents in fact a probability density contour, akin to an atomic orbital [4] [5]. So therefore the best representation that we may hold of the 3-D Universe in its fullness is one that is shapeless and boundless, its best highlevel ensemble descriptor being $\rho_{c o s}$, the equivalent of the quanto-geometric figure of $\sigma^{3}$, both in quality and quantity. The galaxies and galaxy groups that we see pulling away from each other are simply receding from each other just as two electrons constantly recede from each other in an atomic orbital. Their dance is a stochastic dance that does not at all imply the intervention of a presumed anti-gravity dark matter force. There is much more to this specific element of the analysis than intended for this exposition.

I cannot help but conclude that there exists no such thing as a Gaussian distribution in time, no matter what motivation there may be to invent one. The Gaussian distribution is intrinsically stochastic in nature. We have demonstrated why it is so with the Quanto-Geometric axiomatic [3]. The best representation of the 3-D universe is one that considers the Shelling taking place from its most affine and irreducible scale to the largest observable structures, as portrayed in Fig.1, which are tangible and real, and not the fabrication of timeevolution story line along a 15 billion-year course in unnatural time units nowhere to be found, all of which is imaginary and hypothetical, thus fictitious.

\section{No Time Period}

In the light of all of the above, one is in good rights to raise the following question: if the universe is devoid of time, why does time appear to reasonably work for us at our scale?

One of the main reasons why time works for us is that the entire set of phenomena that human life spans consist of is circumscribed within natural transformation groups of rotational symmetry or closed manifolds. Because these forms of symmetry are poor, with only one or two degrees of freedom, prediction on the motional line is equivalent to repeat. Therefore we can create expectation values for motion which can be rigidly described by a sense of timeflow at a certain rate or a time-driven equation. On the grand scale of motion in totalitarian space from rest to $c$, time is only good for the description of action over two most affine points on that scale. On three affine points, which represents parabolic symmetry, the manifold is already open, and action is no longer predictable because choice already emerges in terms of election between a one-two pointer and a one-tree pointer. That a bound electron in an atomic orbital can be described by a classical equation with the time variable on two uttermost affine points of motion is true. But beyond, the equation will fail, because the momentum of the electron becomes unpredictable and can only be captured by statistical account. Same holds true for us at the scale of the Universe stretching much beyond our habitat.

Considering an individual running downhill, a close observer could probably visualize a good equation that would tell when he will get down the hill; the observed protagonist 
would have then behaved just like a rock rolling down the same hill. But he could have decided to take suddenly a diagonal path, or the rock could suddenly break up into two pieces despite the odds. To good approximation, these are unpredictable events that a classical equation will not properly capture entirely. The closer the observer is to you or the rock causally, the best time-driven equation he can write. The farther the observer is to you and the rock, the more likely a stochastic approach will deliver better results to him in terms of dynamical expectation values.

So time seems to work for us principally because we have limited mobility throughout a rather small and slow habitat.

\section{Evolution of the Universe Viewed Thru Quanto-Geometric Quintessence}

The false idea of a Universe in expansion, from wrong observational interpretations of a dynamic cosmos, has led to the question of whether the Universe will expand forever or tend to shrink. A model has been built to support this view from the data gathered about the vacuum energy density based on luminosity of distant supernovae (Fig 5).

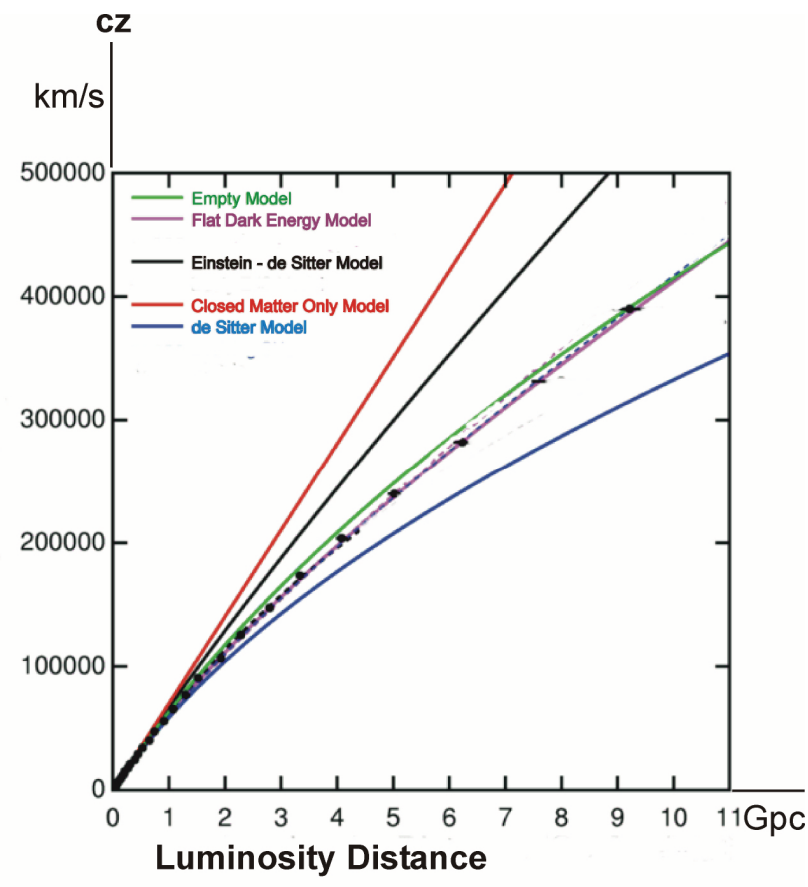

Fig. 5 Evolution of the 3-D Universe projected from vacuum energy density reflected in supernova luminosity, in different cosmological models

In fact there are in existence a number of models of possible evolutionary trends for the Universe (Fig.5). All of them cater however to the idea of time-driven evolution of the
Universe, on the basis of a critical value of the vacuum energy density estimated at:

$$
\rho_{c r i}=0.6 \cdot 10^{-29} \mathrm{~g} / \mathrm{cm}^{3}
$$

These models also promote a figure of cosmological constant $\Omega_{\wedge}$ relating the critical density to the observed, measured or theorized density $\rho_{\text {vac }}$, according to:

$$
\Omega_{\Lambda}=\frac{\rho_{v a c}}{\rho_{c r i}}
$$

The recent luminosity data as of 2015 confirms what previous decade-long data collection had suggested in terms of the closeness of the vacuum energy density to the critical limit:

$$
\begin{aligned}
& \Omega_{\Lambda}=\frac{\rho_{v a c}}{\rho_{c r i}}=0.75 \text { or } \\
& \rho_{v a c}=0.75 \rho_{c r i}
\end{aligned}
$$

The interesting conclusion reached by these models is that if we put the vacuum energy density and ordinary matter density on the same $\Omega$ scale, and further combine them in $\left[\Omega_{\mathrm{M}}+\Omega_{\Lambda}\right]$, then the Universe must be:
1. Open if $\rightarrow\left[\Omega_{\mathrm{M}}+\Omega_{\Lambda}\right]<1$
2. Closed if $\rightarrow\left[\Omega_{\mathrm{M}}+\Omega_{\Lambda}\right]>1$
3. Flat if $\rightarrow\left[\Omega_{\mathrm{M}}+\Omega_{\Lambda}\right]=1$

This characterization is in high match with QuantoGeometric Quintessence [1] [3] whereby covariance between scalar density and associated vacuum spread or space orchestrates three grand moments or phases of the Grand Eigenfunction, whether in one-space or two-space as evidenced by its first-order derivative:

- $\left.\frac{\partial q}{\partial t}\right|_{m}>1$ and $\left.\frac{\partial q}{\partial m}\right|_{t}>1$ in 2-space, or $\frac{d q}{d s}>1$ in one-space, a sector of the graph of the Function where the scalar is predominant over its space partner.

- $\left.\frac{\partial q}{\partial t}\right|_{m}<1$ and $\left.\frac{\partial q}{\partial m}\right|_{t}<1$ or $\frac{d q}{d s}<1$, a sector of the graph where the space tenet is predominant over its scalar partner. 
- $\left.\frac{\partial q}{\partial t}\right|_{m}=1$ and $\left.\frac{\partial q}{\partial m}\right|_{t}=1$ or $\frac{d q}{d s}=1$, a region of the graph where scalar and space tenets are of equated influence on one another.

The $m$ and $t$ variables stand for the two independent space variables of the 2-variable Eigenfunction whereas the $s$ variable stands for the one independent variable of the single variable version of the Eigenfunction (Fig. 6).

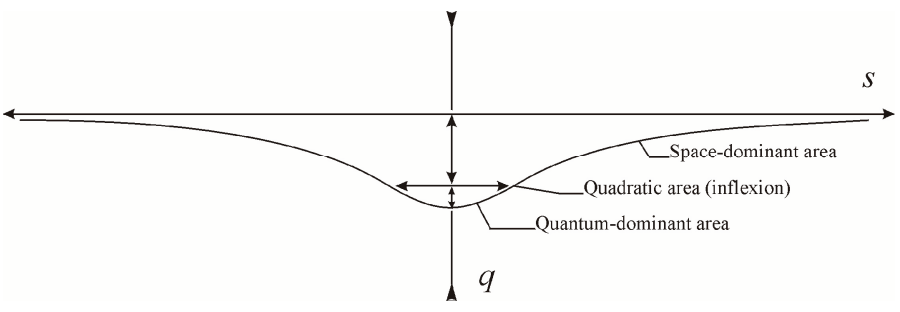

Fig. 6 Quintessence in Quanto-Geometry

Should we use the Quanto-Geometric characterization to interpret the quantum relativistic view offered above, we would state that when the scalar density overwhelms the "vacuum energy density", the Universe must be closed, that is to say adopts a closed shape which is any of spherical, ellipsoidal or even parabolic morphology. But if the "vacuum energy density" overwhelms scalar density of the Universe, then it will adopt an open shape, that is to say any of the 5 gradients of hyperboloid morphology. And if the "vacuum energy density" is to be of equal covariant weight with scalar density, then the universe will be flat, which means that it will adopt the morphology proper to a 2-space hyperbola. We caution and clarify that these shapes to the universe are not real exogenous shapes but virtual endogenous. And as such, unobservable.

What essentially distinguishes our view in QuantoGeometric Theory from this quantum relativistic view is that the 3-D World plane is not to adopt one or the other of these three courses of evolution, but that it in fact develops these three phases simultaneously because the scalar-space distribution in the arena is a stochastic distribution in accord with the Grand Eigenfunction. If one were to ask the question as to the reason why the quantum relativistic hypothesis of 3 possible phases is valid, the answer will have to be that it is so because behind this tri-partite course is the stochastic Grand Eigenfunction with a preeminent Tri-Valent quantum-space covariance at its heart. One could also add as a corollary to the answer that it is also because the value of the vacuum energy density that sustains the quantum relativistic view is in fact the cube of the standard deviation parameter $\left(\sigma^{3}\right)$ of the Grand
Eigenfunction. End of story. And end of life to the time-driven narrative.

\section{Why the Quantum Relativistic Big Bang View is Incomplete and Distorting}

Let me make this as clear as I possibly can: you cannot have a view of the Universe in terms of the dynamics of the large-scale structures without taking into account in the same description the infinitesimal structures, not how they've been in the past but how they behave NOW. Else the description is incomplete and disjoint. In the absence of a Shell based description that can explain why the 3-D universe is structured in Shells and the nature of the symmetries governing this development as well as how they give rise to the fundamental constants or invariants sustaining those Shells, one has not a true physical description, but a hypothetical story. It is not possible to hold within the same view 4 or 5 different and incoherent approaches to the same fundamental question of the state of the vacuum, because they negatively reflect on the overarching perspective which is the quantum relativistic picture of a Big Bang universe so proven to misunderstand the vacuum.

What is wrong with this view is principally what is missing in it. The Standard Model has not understood the quantum vacuum and its deployment in Shells at its scale. The notion of dimensional time at that scale, an estranged concept in the original quantum theory, has distorted and falsified all possibilities to gain insight into the inner dynamics of the vacuum. Is the vacuum $\rho_{\text {hep }}$ indictment not to mean: scratch and start over? Inarguably so.

\section{Conclusion}

F. Wilczek's assessment of the vacuum crisis in 2001 and its implications for modern physics constitutes the most lucid observations ever made concerning this thorny problem, in my humble view. His assessment is very much a call for new physics that is not in the direction of offering extensions to the Standard Model, but an entirely new framework for physical science. He radically and rightly thought that the scope of the crisis demanded that much. What he may not have realized, however, is that the call for new physics in the wake of this dramatic failure causing a quagmire to the entire science indeed intersects with D. Hilbert's call for axiomatization of physics issued since early $20^{\text {th }}$ century. It is not possible to overcome this crisis in fundamental physics without uncovering the origin of the primordial symmetries in nature and how they develop the fundamental physical constants as pillars of the entire physical world. This mathematical-physics enterprise, long overdue in physics, is 
the only one that can provision the badly needed new framework. It is our hope that the Quanto-Geometric Theory is in effect providing this new window on the horizon.

\section{REFERENCES}

[1] Jean-Claude, J. Joseph, Quanto-Geometry: Overture of Cosmic Consciousness and Universal Knowledge for All, Volume I, Quantometrix, 2015

[2] Jean-Claude, J. Joseph, Quanto-Geometry: Overture of Cosmic Consciousness and Universal Knowledge for All, Volume II, Quantometrix

[3]Jean-Claude, J. Joseph, Quanto-Geometric Tensors \& Operators on Unified Quantum-Relativistic Background, Academia.edu, November 2016 - Research Gate, 2017

[4] ACOSTA, V., and COWAN, C., and GRAHAM, B.J., Essentials of Modern Physics, Harper \& Row Publishers, 1973

[5] Cruz, Andoni, Chamizo, Jose, Garritz, Diana, Estructura Atómica: Un Enfoque Químico, Fondo Educativo Interamericano, 1986

[6] Robert L. Oldershaw, Towards A Resolution Of The Vacuum Energy Density Crisis, arXiv:0901.3381, 2009

[7] Asher Peres, Critique of the Wheeler-Dewitt Equation, arXiv:gr-qc/9704061, 1997

[8] George E. A. Matsas et al, The Number of Dimensional Fundamental Constants, arXiv:0711.4276v2, 2007

[9] John C. Baez, Emory F. Bunn, The Meaning of Einstein's Equation, E.F.B., 2006

[10] Edward L. Wright, Vacuum Energy Density, How can Nothing Weigh Something, UCLA, 1998-2012

[11] Erik Morgan, Estimating the Vacuum Energy Density - an Overview of Possible Scenarios, Jozef Stefan Institute, 2005

[12] David Hilbert, Mathematical Problems - Lecture delivered before the International Congress of Mathematicians at Paris, 1900 Bulletin of the American Mathematical Society 8 (1902), p. 437-479.

[13] Hooft Gerard't: The Conceptual Basis of Quantum Field Theory, Institute for Theoretical Physics, Utrecht University and Spinoza Institute, 2004

[14] Young, Nicholas, An Introduction to Hilbert Space, Cambridge University Press, 1988
[15] Donald H. Perkins, Introduction to High-Energy Physics, Addison-Wesley, 1982

[16] David J. Gross, The Role of Symmetry in Fundamental Physics, National Academy of Science, PNAS vol. 93 no. 25 14256-14259

[17] S.L. Wymer Meardon, The Elements of Fiber Optics, Regents/Prentice Hall, 1993

[18] D. Halliday, R.Resnick, J. Walker, Fundamentals of Physics, $5^{\text {th }}$ Edition, John Wiley \& Sons, 1997

[19] Howard P. Layer, Length - Evolution from Measurement to Fundamental Constant, Precision Engineering Division of the Manufacturing Engineering Lab at NIST

[20] S.S. Stepanov, Fundamental Physical Constants and the Principle of Parametric Incompleteness, arXiv:physics/9909009v1, 1999 